\title{
Report of the 14th International Workshop on Aspect-Oriented Modeling
}

\author{
Jörg Kienzle ${ }^{1}$, Jeff Gray ${ }^{2}$, Dominik Stein ${ }^{3}$, Thomas Cottenier ${ }^{4}$, \\ Walter Cazzola ${ }^{5}$, and Omar Aldawud ${ }^{6}$ \\ ${ }^{1}$ McGill University, Montreal, Canada \\ ${ }^{2}$ University of Alabama, Tuscaloosa, USA \\ ${ }^{3}$ University of Duisburg-Essen, Essen, Germany \\ ${ }^{4}$ UniqueSoft, Palatine, USA \\ ${ }^{5}$ University of Milan, Italy \\ ${ }^{6}$ Illinois Institute Of Technology, Chicago, USA \\ Joerg.Kienzle@mcgill.ca, gray@cs.ua.edu, dominik.stein@icb.uni-due.de, \\ cotttho@gmail.com, cazzola@dico.unimi.it, aldaoma@iit.edu
}

\begin{abstract}
This report summarizes the outcome of the 14th Workshop on Aspect-Oriented Modeling (AOM), as part of the 12th International Conference on Model Driven Engineering Languages and Systems - MoDELS 2009 - in Denver, Colorado, USA, on the 4th of October 2009. The workshop brought together researchers and practitioners from two communities: aspect-oriented software development (AOSD) and software model engineering. This paper gives an overview of the accepted submissions, and summarizes the results of the discussion.
\end{abstract}

\section{Introduction}

This paper summarizes the outcome of the 14th edition of the successful AspectOriented Modeling Workshop series. An overview of what happened at previous editions of the workshop can be found at http://dawis2.icb.uni-due.de/aom/. The workshop took place at the Hyatt Tech Center Hotel in Denver, Colorado, USA, on Sunday, October 4th 2009, as part of the 12th International Conference on Model Driven Engineering Languages and Systems - MoDELS 2009.

Participation to the workshop was open to anyone attending the conference, and as a result there were approximately 30 participants. A total of 5 position papers were submitted and reviewed by the program committee, all of which were accepted to the workshop.

The rest of this report is structured as follows: Section 2 gives an overview of the presentations of the accepted papers that took place in the morning sessions. Section 3 summarizes the presentations on the crisis management system case study of the first afternoon session. Section 4 gives an overview of the questions and comments that were voiced in the second afternoon session. Section 5 concludes the report. 


\section{Overview of Presentations}

Gefei Zhang from the Ludwig-Maximilians-Universität München in Germany presented the paper "HiLA: High-Level Aspects for UML State Machines". State machines are popular, simple and intuitive for modeling the behavior of reactive systems. However, Gefei pointed out that state machines face important modularity problems when used to model behavior at a high level of abstraction. To address this problem, he proposed HiLA. The language provides three different kind of aspect models that allow a modeler to separate behavior pertaining to a different concern: synchronization aspects, history-based aspects, and low-level transformation aspects. To illustrate the approach, Gefei presented a simple state machine describing the behavior of a guard in a game environment that is composed of 3 rooms. He then showed how to extend the model to add more detailed behavior to the guard (e.g., to prevent the guard from moving from one room to the other in certain conditions, or to inhibit the guard from fighting in a specific room). This additional behavior was modeled in separate HiLA aspect models, and did not require the base model to be modified. HiLA also provides features that allow aspects to model behavior that is triggered by specific state transition sequences in the base, support for interference detection, and are model-checkable.

Wisam Al Abed from McGill University in Montreal, Canada, presented how the Reusable Aspect Models approach, previously published in [12] can be extended to support information hiding principles. In object-orientation, the class construct allows a modeler to encapsulates state and behavior. An interface allows a modeler to expose some of the encapsulated state and behavior to the outside world; everything else remains hidden within the class. Wisam argued that aspect-orientation adds a new dimension to encapsulation, allowing a modeler to group model elements related to the structure and/or behavior of a given concern together, even if the concern's state and behavior crosscuts the class boundaries. As a result, information hiding becomes tricky. Wisam proposed that in addition to a public interface that exposes the state and behavior that the aspect is designed to provide to the outside world, model elements within an aspect need to define an intra-aspect interface that exposes state and behavior to the model elements within the same aspect. In addition, in order to support aspect hierarchies, an aspect weaver should automatically remove the public interface of an aspect during the weaving process by moving the operations from the public interface to the intra-aspect interface. As a result, aspects give the developer the power to hide design decisions effectively, even those that require the interaction of instances of multiple classes.

Jorge Barreiros from the Instituto Politécnico de Coimbra in Portugal presented "Integrating AORA to the Problem Frames Approach". He highlighted the fact that problem frames do not systematically address the problem of separation of crosscutting concerns. He explained how it is possible to integrate the meta-model of the Problem Frames (PF) approach with the meta-model of the Aspect-Oriented Requirements Analysis (AORA) approach with the aim of making PF analysis models more modular. As a result, an AORA concern can 
detail the requirements which must be fulfilled by a PF machine's for a specific problem. AORA responsibilities can then be mapped to interface specifications of problem frame machines. He suggested the following process: 1) design the context diagram to structure and delimit the problem (PF); 2) Identify the main concerns following AORA; 3) Generate AORA templates for each concern; 4) Design a problem diagram for each functional concern (PF); 5) Identify match points and crosscutting concerns; 6) Specify composition rules using AORA. Jorge illustrated the approach with the car parc case study.

Valter Camargo from the Federal University of Sao Carlos in Brazil presented his paper entitled "A Preliminary Comparative Study Using UML-AOF - a UML Profile for Aspect-Oriented Frameworks". UML-AOF, which was proposed in a previous AOM workshop [3], is a UML profile that provides stereotypes that allow a modeler to better document the instantiation of an aspect-oriented framework within a target model, as well as provide the developers of an aspectoriented framework features that facilitate the maintenance activity. Valter first presented a review of the UML-AOF stereotypes, and then continued on to describe an empirical study that was conducted in a university setting to compare ML-AOF with Evermann's profile [4, which is a UML profile that was developed to describe aspect-oriented designs of programs implemented with AspectJ. In the empirical study, 8 graduate students divided into two groups were asked to look at code of two case study systems, a hotel reservation system and workshop management system. Two crosscutting concerns, persistence and security, were implemented in both systems using an aspect-oriented framework. The students were given a questionnaire, in which they had to answer questions about 1) the idioms and patterns used to link the AOF with the base application; 2) extension points of the AOF; 3) questions that asked them to determine if a certain class or interface belonged to the base application or the AOF; 4) aspect-oriented programming language specific questions.

The last presentation entitled "Reusable Model Slices" was also given by Jorge Barreiros from the Instituto Politécnico de Coimbra in Portugal. Reusable model slices provide features that allow the modeler to express method signature manipulations that are to occur when a reusable model slice is woven into a base model. The presented approach also allows one aspect model to specialize another aspect model. Two kinds of specialization are supported: incremental and partial. Incremental specialization adds new template parameters to a model, while partial specialization fixes one of the template parameters. When specifying element creation within an aspect, the approach offers scoping features to specify if the created model element is to be universal to the target model (i.e. a single instance of the element is created), global (i.e. one instance of the element is created for each package), or local (i.e. one instance is created for each parameter binding).

\section{Crisis Management System Case Study Session}

The afternoon session from 2:00 - 3:30 was devoted to a discussion on the crisis management system (CMS) case study. The CMS case study was proposed in [5] 
as a common case study for the aspect-oriented modeling research community. A crisis management systems is a system that helps in identifying, assessing, and handling a crisis situation by orchestrating the communication between all parties involved in handling the crisis, by allocating and managing resources, and by providing access to relevant crisis-related information to authorized users. The case study description contains informal requirements of crisis management systems in general, a feature model for a CMS product line, use case models for a car crash CMS (CCCMS), a domain model for the CCCMS, an informal physical architecture description of the CCCMS, as well as some design models of a possible object-oriented implementation of parts of the CCCMS backend. AOM researchers that want to demonstrate the power of their AOM approach or technique can hence apply their approach to whatever part of the case study is the most appropriate to illustrate their ideas.

An open call of the journal Transactions on Aspect-Oriented Software Development published by Springer Verlag sollicitating submissions presenting aspectoriented models of the CMS for a special issue of the journal was released in May 2009. Jörg Kienzle, the guest editor of this special issue and co-organizer of this workshop, received 11 paper submissions responding to the call in September 2009. Authors of 6 of the papers were actually attending the AOM workshop in Denver, and agreed to give a short presentation describing how their AOM approach was applied to the CMS.

Gunter Mussbacher from the University of Ottawa, Canada, showed aspectoriented requirements models of the CMS expressed using the aspect-oriented user requirement notation (AoURN), which consists of aspect-oriented use case maps and the aspect-oriented goal-oriented requirements language. He identified aspects such as coordination, strategy recommendation, resource management and communication. Gefei Zhang from the Ludwig-Maximilians-Universität München in Germany presented how he modeled parts of the CMS using HiLA, the aspect-oriented modeling approach he had presented in the morning. In particular, he presented models of the login aspect, and explained how modelchecking can be applied to HiLA moels to verify desired system properties. Frans Sarnen from the Katholieke Universiteit Leuven, Belgium, pointed out the pointcut fragility problem, and subsequently described a software development process that leads a developer to identify crosscutting concerns at the requirements level within use cases. Then, stable domain abstractions are discovered, which can be used within architectural design, and as basic building blocks for constructing pointcut interfaces that are unlikely to change when the software evolves. Sebastien Mosser from the university of Nice, France, presented how ADORE, an aspect-oriented technique for composition of orchestrations for executable business processes, was used to design the workflows of the crisis management system. He presented a process in which a design model of service orchestrations is extracted from the use cases of the requirements document. Florian Heidenreich from Dresden University in Germany showed a comparison of two different approaches, FeatureMapper and VML*, that allow a developer to map feature models to other models of a product 
line. To properly illustrate the product line development and to highlight the advantages and disadvantages of both approaches, he introduced an additional specific crisis management system product: a flood crisis management system. Jörg Kienzle from McGill University in Canada showed how the Reusable Aspect Models (RAM) approach was applied to the design of the car crash crisis management system. He explained how the design of the CreateMission functionality was decomposed into a hierarchy of 23 interdependent aspect models.

\section{Summary of the Discussion Groups}

The second afternoon session provided an opportunity for open discussion among the participants. Those who wanted to voice a position or ask a question first expressed their comments to the whole group. The set of questions and positions were collected and approximated clusters of topics were formed. The group as a whole then considered each cluster and discussed each topic. A summary of the list of topics and pertinent points stemming from the discussion follows.

Verification of aspect correctness: A common theme among the concerns expressed by attendees related to the important issue of syntactic and semantic verification of the composition of multiple aspects. The topic of aspect composition and the associated correctness is not a new concern, but attendees discussed how many of the proposed AOM approaches could be tested (e.g., case studies, metrics). Several attendees wanted to know about the potential for metrics that might help guide the reuse of aspects. A specific domain of interest that was brought up in the discussion was the interest in security aspects and the desire to have some confidence in the correctness of their composition with a base application.

AOM for managing complexity: The increased demands of software capabilities raised concerns over the management of aspect complexity, as well as base system complexity. AOM promises reduced complexity by more advanced separation of concerns. However, managing large sets of aspects, aspect dependencies and interactions, and correct weaving of complex models can be a challenge. Attendees questioned whether we have mature enough approaches and tools to leverage reduced complexity using AOM approaches. A discussion of aspect hierarchies then ensued with comments on how to compose new aspects from higher level aspects, and how to extend and specialize aspects. The reuse of aspects led to some comments about the desired level of granularity of the aspect and how to extract composition rules to help with variations in granularity. The evolution of aspects was also a focus of many workshop participants within the context of complexity concerns. The complexity of AOM and aspects in general were discussed in the context of aspect evolution (e.g., base model changes, aspect changes, sub-aspect changes amid those aspects that were composed from other higher level aspects).

Application niche areas: A category of niche areas for AOM application was discussed. AOM@Runtime was a popular concept for dialogue among the 
participants. Many attendees wanted to know how to use AOM techniques for implementing dynamic variability. Within this context, there were several concerns about the performance of AOM weavers and the need for efficiency in the model weaving. Given the strong focus on UML in the AOM area, there were questions about the use of aspects in the growing area of domain-specific modeling languages. The overall state of maturity amid AOM tools was mentioned; the lack of downloadable tools amid a plethora of "paper-based" concept ideas highlighted the need for more implementation focus for AOM.

\section{Concluding Remarks}

The organizers for this edition of the workshop were Omar Aldawud, Walter Cazzola, Thomas Cottenier, Jeff Gray, Jörg Kienzle, and Dominik Stein. An expert program committee provided assistance in reviewing the submitted papers. The members of the program committee were Mehmet Aksit, Aswin Van den Berg, Frank Fleurey, Sudipto Ghosh, Stefan Hanenberg, Phillipe Lahire, Funter Mussbacher, Alfonso Pierantonio, Ella Roubtsova, Pablo Sánchez, Befir Tekinerdogan, Julie Vachon, Markus Völter, Jon Whittle, and Steffen Zschaler. Last but not least, we'd like to thank all submitters and participants of the workshop who contributed with their papers and positions.

\section{References}

1. Klein, J., Kienzle, J.: Reusable Aspect Models. In: 11th Aspect-Oriented Modeling Workshop, Nashville, TN, USA, September 30 (2007)

2. Kienzle, J., Abed, W.A., Klein, J.: Aspect-Oriented Multi-View Modeling. In: Proceedings of the 8th International Conference on Aspect-Oriented Software Development - AOSD 2009, March 1-6, pp. 87-98. ACM Press, New York (2009)

3. Júnior, J.U., Camargo, V.V., Chavez, C.V.F.: UML-AOF: a profile for modeling aspect-oriented frameworks. In: AOM 2009: Proceedings of the 13th workshop on Aspect-Oriented Modeling, pp. 1-6. ACM, New York (2009)

4. Evermann, J.: A meta-level specification and profile for aspectj in UML. In: AOM 2007: Proceedings of the 10th international workshop on Aspect-oriented Modeling, pp. 21-27. ACM, New York (2007)

5. Kienzle, J., Guelfi, N., Mustafiz, S.: Crisis Management Systems - A Case Study for Aspect-Oriented Modeling. Technical Report SOCS-TR-2009.3, McGill University, Montreal, Canada (2009) 